\title{
Relativistic jet production from accreting compact objects
}

\author{
H.C. Spruit* \\ Max-Planck-Institiut für Astrophysik, Postfach 1317, D-85741 Garching \\ E-mail: henk@mpa-garching.mpg.de
}

\begin{abstract}
The magnetic theory for the production of jets by accreting objects is summarized with attention to some outstanding problem areas. An effort is made to show the connections behind the somewhat diverging nomenclature in the literature, and to contrast the different points of view about basic mechanisms. A key problem is the acceleration of the jet flow to high Lorentz factors. In this context, the role of dissipation of magnetic energy in accelerating the flow is discussed, the importance of which has been realized only recently. A second theme is the 'collimation problem', discussion of which is hampered by incorrect ideas about the role of 'hoop stress'. Current views on these problems are still strongly biased by the restriction to 2 dimensions (axisymmetry) in previous analytical and numerical work. Estimates are given how jets will behave differently in the 3-dimensional world. An interesting problem area is the nature and origin of the strong, preferably highly ordered magnetic fields known to work best for jet production, and observational evidence for such fields. I argue that the presence or absence of such fields may well be the 'second parameter' governing the X-ray spectra and timing behavior in X-ray binaries.
\end{abstract}

VI Microquasar Workshop: Microquasars and Beyond

September 18-22 2006

Società del Casino, Como, Italy

\footnotetext{
* Speaker.
} 


\section{The standard magnetic acceleration model}

The magnetic model has become the de facto standard for explaining (relativistic) jets, that is, collimated outflows. In part this has been a process of elimination of alternatives, in part it is due to analytic and numerical work providing a sound theoretical basis for some essential aspects of the mechanism. It should be remembered that a key observational test of the model is still largely missing. Evidence for magnetic fields of the configuration and strength required by the model is indirect at best. Magnetic fields are detected indirectly through synchrotron radiation (such as the radio emission of extragalactic jets), and in some cases directly through the Zeeman effect in spectral lines $\left(\mathrm{OH}\right.$ or $\mathrm{H}_{2} \mathrm{O}$ masers) in young stellar objects and protoplanetary nebulae (e.g. Hutawarakorn et al. 2002, Bains et al. 2004, Vlemmings et al. 2005). Most of these detections, however, do not refer to the inner regions of the flow where most of the magnetic action is expected to take place.

In the standard magneto-centrifugal acceleration model for jets produced by an accretion disk (Bisnovatyi-Kogan and Ruzmaikin 1976, Blandford and Payne 1981) there are three distinct regions. The first is the accretion disk, where the rotating field lines are anchored; here the kinetic energy of rotation (perhaps also the gas pressure) dominates over the magnetic energy density. As a result, the field lines corotate with the disk in this region.

Assuming the disk to be cool, the atmosphere of the disk has a low density and gas pressure. In this region, the magnetic field dominates, so that it must be approximately force-free $(\nabla \times \mathbf{B} \times$ $\mathbf{B}=0$ ), like the magnetic field in much of the solar atmosphere. It forces the flow of gas into corotation, with only the velocity component along the field unrestricted by magnetic forces. The flow experiences a centrifugal force accelerating it along the field lines, much as if it were carried in a set of rotating rigid tubes anchored in the disk. This acceleration depends on the inclination of the field lines: there is a net upward force along the field lines only if they are inclined outward at a sufficient angle. Field lines more parallel to the axis do not accelerate a flow. The conditions for collimation and acceleration are thus somewhat opposite to each other, in the magnetocentrifugal acceleration picture.

If acceleration by the centrifugal mechanism is effective, something is needed in addition, after the acceleration, to collimate the flow to the observed narrow opening angles of jets (section 6). It is conceivable that this does not happen in all cases: less collimated flows may also exist. They would be harder to detect, but have already been invoked for observations such as the 'equatorial outflows' in SS433 (see R. Spencer, this volume).

The transfer of energy powering the outflow is thus from gravitational energy to kinetic energy of rotation, and from there to kinetic energy of outflow via the magnetic field. Note that in the centrifugal picture the magnetic field plays an energetically passive role: it serves as a conduit for energy of rotation, but does not itself act as a source of energy. The function of the magnetic field in the acceleration process can also be viewed in a number of different ways; this is discussed further below.

The magnetic field strength decreases with distance and the flow speed increases due to the centrifugal effect. The kinetic energy density becomes comparable with the magnetic energy density at the Alfvén radius, $r_{\mathrm{A}}$. Around this point the assumption that the field lines corotate with their anchoring points stops being valid. Centrifugal acceleration stops around this point, and the field 
lines start lagging behind, with the consequence that they get 'wound up' into a spiral. Beyond the Alfvén radius, the rotation rate of the flow gradually vanishes since it continues to expands away from the axis, while the flow speed approaches its asymptotic value. If nothing else were happening, the field in this region would thus be almost purely azimuthal, with one loop of azimuthal field being added to the flow for each orbit of the anchoring point. In fact, this state is very unlikely to survive for much of a distance beyond $r_{\mathrm{A}}$, because of other (3-dimensional) things actually happening.

When the centrifugal picture applies, most of the (logarithmic) increase in flow speed takes place inside the Alfvén radius. The transfer of magnetic to kinetic energy takes place effectively around the Alfvén radius.

\section{Length scales}

The energy release powering a relativistic outflow happens near the black hole, say $10^{7} \mathrm{~cm}$ in the case of a microquasar. The narrow jets of microquasars seen at radio-wavelengths appear on scales of the order $10^{17} \mathrm{~cm}$. In other words, on scales some ten orders of magnitude larger. It is quite possible that some of jet properties are determined on length scales intermediate between these extremes, at least in some cases. In Spruit et al. (1997), for example, we have argued that collimation of the flow may actually take place on scales large compared with the Alfvén radius, at least in very narrow jets. In the following, I will show that such intermediate length scales can also be crucial for acceleration to high Lorentz factors, in addition to the region around the Alfvén radius that plays the main role in the axisymmetric centrifugal acceleration process.

Much of the current thinking about the processes of launching, acceleration and collimation of the jet is based on previous analytical models. Numerical simulations of magnetic jets are now becoming increasingly realistic and useful. They are, however, quite restricted in the range of length scales and time scales they can cover. This leads to a bias in the interpretation of such simulations: the tendency is to assume that all steps relevant to the final jet properties happen within the computational box. This bias will probably last as long as simulations covering realistic ranges in length and time scale are impossible.

\section{Magnetic jets}

\subsection{Power sources, composition of the jet}

Jets powered by the rotation of a black hole (Blandford-Znajek mechanism) are often assumed to consist of pair plasmas, while outflows from rotating disks are regarded as consisting of a normal ion-electron plasma. These associations are not exclusive, however. The presence of a magnetic field threading the hole requires the presence of an accretion disk to hold it in place. Hence it is quite likely that (part of) the jet accelerated by the hole is actually fed with mass accreting from the disk rather than a pair plasma generated in situ. The simulations by De Villiers et al. (2005), McKinney and Gammie (2004) are examples of this.

The opposite may also happen. A strong field threading a thin (cool) disk will not be easily loaded with mass from the disk unless the field lines are sufficiently inclined outward, away from the vertical (Blandford and Payne, 1981). In addition, the mass loading decreases with increasing 
field strength, for a given field line geometry (Ogilvie and Livio, 2001). In the absence of mass loaded on the field lines, the rotating vacuum field may produce a pair plasma, by processes like those thought to produce the relativistic pair plasma outflows from pulsars. This case has not received much attention so far.

In the literature, the phrase 'Poynting flux' is often associated specifically with relativistic and/or pair-dominated flows. It applies quite generally, however: equally to relativistic and nonrelativistic flows, and independent of their composition.

\section{2 'Centrifugal' vs. 'magnetic' vs. 'Poynting flux'}

The physical description of the flow-acceleration process has been a source of confusion. There are alternatives to the centrifugal picture sketched above: descriptions in terms of magnetic forces or in terms of 'Poynting flux conversion'. These descriptions are largely equivalent, however; which one to take is a matter of personal taste, or the particular aspect of the problem to be highlighted. For example, the term 'Poynting jet' is sometimes used. It does not refer to a different mechanism, however, but rather to a particular point of view of the same process.

In a frame of reference corotating with the anchoring point of a field line, the flow is everywhere parallel to the magnetic field (e.g. Mestel, 2001). The component of the Lorentz force parallel to the flow vanishes in this frame. There is no magnetic force accelerating the flow, instead, in a rotating frame the role of the Lorentz force is taken over by the centrifugal force. This is sometimes viewed as a contradiction for a magnetic model of acceleration: how can one still call the acceleration magnetic if there is no work done by magnetic forces?

If, on the other hand, the same process is evaluated in an inertial frame, the centrifugal force is absent. Instead, one finds that the flow is accelerated by a force associated with the azimuthal component of the magnetic field: $\mathbf{F}=\nabla B_{\phi}^{2} / 8 \pi-B_{\phi}^{2} /(4 \pi \varpi)$, where $\varpi$ is the distance from the axis. The two descriptions, magnetic and centrifugal, are mathematically equivalent, being related by a simple frame transformation. The centrifugal picture is an elegant way to visualize the acceleration as long as the magnetic field lines corotate with their anchoring point. Where they do not corotate, the field gets wound up into a predominantly azimuthal field, and the acceleration is described most simply in terms of the forces exerted by the azimuthal field component $B_{\phi}$.

Finally, the same process can also be viewed as a conversion of a Poynting flux of electromagnetic energy into kinetic energy. To see this, recall that in magnetohydrodynamics the electric field $\mathbf{E}$ is given by $E=\mathbf{v} \times \mathbf{B} / c$, so that the Poynting flux

$$
\mathbf{S}=\frac{c}{4 \pi} \mathbf{E} \times \mathbf{B}
$$

can be written as

$$
\mathbf{S}=\mathbf{v}_{\perp} B^{2} /(4 \pi),
$$

where $\mathbf{v}_{\perp}$ is the component of the flow velocity perpendicular to the magnetic field. This can be interpreted as a flux of magnetic energy, advected with the fluid, in a direction perpendicular to the field lines ${ }^{1}$.

\footnotetext{
${ }^{1}$ The actual flux of magnetic energy would of course be $\mathbf{v}_{\perp} B^{2} / 8 \pi$. The 'missing' $B^{2} / 8 \pi$ represents the ' $P d V$-work' done by the source of the flow against the magnetic pressure at the base of the flow, c.f. section 5 .
} 
Borrowing a close analogy from hydrodynamic flows, the Poynting flux plays the role of a 'magnetic enthalpy flux'. The centrifugal acceleration process is equivalent to the gradual (and incomplete) conversion of Poynting flux into a flux of kinetic energy, much like the conversion of enthalpy into kinetic energy in an expanding hydrodynamic flow. Near the base of the flow (for example the surface of the accretion disk which supplies the mass flux into the wind), the enthalpy flows almost entirely in the form of a Poynting flux $\mathbf{S}$. $\mathbf{S}$ declines gradually with distance and the kinetic energy increases correspondingly, most of the energy transfer taking place around the Alfvén radius (in the axisymmetric case, see however section 5).

\subsection{Poynting flux conversion efficiency: axisymmetric}

Since the flow is magnetic everywhere, at least some of the energy is carried in the form of a magnetic energy flux. The work done by the central engine therefore is not converted completely into kinetic energy, and one may wonder what determines the efficiency of conversion of Poynting flux.

It turns out that the answer depends critically on the symmetry of the flow. When 3-D, nonaxisymmetric processes are allowed, conversion can be much more efficient than in axisymmetric flows. This is discussed further in section 5. Since much of the current views are still based on axisymmetric models, however, consider these first.

If $S_{0}$ is the Poynting flux at the base of the flow (equal to the power output of the central engine) and $F_{\mathrm{K}}$ the kinetic energy flux, we can define this efficiency $f$ as

$$
f=F_{\mathrm{K} \infty} / S_{0}=F_{\mathrm{K} \infty} /\left(F_{\mathrm{K} \infty}+S_{\infty}\right),
$$

where ${ }_{\infty}$ denotes the asymptotic values at large distance. A simple model for which this can be calculated is the cold Weber-Davis model (Mestel 2001, Spruit 1996). In this model the poloidal field varies as $1 / r^{2}$ (a 'split monopole'), and the gas pressure is neglected. In the nonrelativistic limit, the conversion efficiency in this model is $f=1 /\left(1+(16 / 3)^{1 / 3}\right) \approx 0.36$, so that about $2 / 3$ of the power delivered by the central engine remains in the flow as magnetic energy.

In the relativistic case, i.e. when the flow reaches large Lorentz factors $(\gamma)$, a smaller fraction of the Poynting flux is converted to kinetic energy, in an axisymmetric flow. The relativistic extension of the Weber-Davis model has been given already by Michel (1969), (see also Goldreich and Julian 1970). If $(r, \theta, \phi)$ are spherical coordinates centered on the source of the flow, the simplest case to visualize is a flow near the equatorial plane, $\theta=\pi / 2$. The 'split monopole' assumption for the poloidal field components implies $v_{\theta}=B_{\theta}=0$. As in the nonrelativistic case, the field at large distances is nearly exactly azimuthal. The radial component of the Lorentz force is then

$$
F_{r}=\partial_{r} B_{\phi}^{2} /(8 \pi)-B_{\phi}^{2} /(4 \pi r) .
$$

From the induction equation one finds that

$$
B_{\phi} r v_{r}=\mathrm{cst},
$$

i.e. the flux of azimuthal field lines is constant. Asymptotically for $\gamma \rightarrow \infty, v \approx c$, so $B_{\phi} \sim 1 / r$. The two terms in the Lorentz force then cancel. Eq 3.4 holds at the equator, but one verifies easily that the same cancellation actually applies at all latitudes, in this split monopole configuration. 
The consequence of this cancellation is that the flow stops being accelerated as soon as it develops a significant Lorentz factor. From then on acceleration and conversion of Poynting flux slow down. Moderately efficient conversion of Poynting flux to kinetic energy is possible, but only up to modest Lorentz factors. High Lorentz factors are also possible, but at the price of converting only a small fraction of the energy flux. This is seen in the expression for the terminal Lorentz factor $\Gamma_{\infty}$ in Michel's model:

$$
\Gamma_{\infty} \approx \mu^{1 / 3}
$$

where $\mu$ is Michel's magnetization parameter,

$$
\mu=B_{0}^{2} /\left(4 \pi \rho_{0} c^{2}\right),
$$

and $B_{0}, \rho_{0}$ are the magnetic field strength and mass density at the base of the flow (where it is still non-relativistic). If conversion of Poynting flux into kinetic energy were complete, it would produce a flow with Lorentz factor $\Gamma_{\mathrm{c}}$,

$$
\Gamma_{c}=\mu \text {. }
$$

The actual efficiency of conversion is thus

$$
f \equiv \Gamma_{\infty} / \Gamma_{\mathrm{c}} \approx \mu^{-2 / 3} \approx 1 / \Gamma_{\infty}^{2},
$$

a small number if large Lorentz factors are to be achieved. One gets either good conversion of Poynting flux into kinetic energy, or large terminal speeds but not both.

\subsubsection{Better conversion: diverging flows}

The conclusion from the previous subsection holds under the 'split monopole' assumption that (apart from the azimuthal flow component) the flow expands exactly radially. If this is not the case, the cancellation is not exact, and continued acceleration possible. To achieve this, the magnetic pressure gradient term in (3.4) has to be larger, relative to the second term, than it is in the split monopole geometry. For this to be the case the azimuthal field has to decrease more rapidly with distance. Let $d(r)$ be the distance in a meridional plane between two neighboring field lines. In the split monopole, $d \sim r$. In the absence of processes destroying azimuthal magnetic field (more about this below) the induction equation yields, as generalization of (3.5):

$$
B_{\phi} d(r) v_{r}=\mathrm{cst}, .
$$

Assuming, for example $d \sim r^{\alpha}$, the Lorentz force is, from (3.4):

$$
F_{r}=2(\alpha-1) \frac{B_{\phi}^{2}}{8 \pi r}
$$

For the force to be outward, $\alpha$ must be larger than 1, that is, the field lines have to diverge more rapidly with distance than purely radial. In other words, the flow has to decollimate to accelerate by this mechanism (Phinney 1983, Begelman and Li 1994), perhaps the opposite of what intuition would tell.

How much of an acceleration is possible in this way? A detailed study of the effect of varying flow geometry in the general relativistic case, including the contribution from thermal pressure, 
has been given by Daigne and Drenkhahn (2002). Reasonable conversion efficiencies, of order $50 \%$, are achieved if the opening angle $d / r$ of the flow increases by a factor of order 10 . This is relatively independent of the distance over which this increase takes place, of the inclusion of thermal pressure, and is not modified much by general relativistic effects. It is necessary, however, that the expansion takes place beyond the fast mode critical point; divergence closer in has no effect on the final Lorentz factor. This effect is quite analogous to the case of a hydrodynamic flow (Holzer, 1977), with the fast mode critical point replacing the sonic point of the hydrodynamic flow.

These conclusions appy to each individual 'flow line', i.e. one-dimensionally (ignoring the azimuthal coordinate because of axisymmetry). Thus they apply whether the flow is calculated as a consistent 2-D model, or with an assumption like the 'split monopole' shape for the flow lines.

The limitations of this process of acceleration by divergence of the opening angle become more severe when actual physical conditions leading to divergence are considered. A limiting factor is causality. In a flow of Lorentz factor $\gamma$, parts of the fluid moving at angles differing by more than $\theta_{\max }=1 / \gamma$ are causally disconnected: there is no physical mechanism that can exchange information between them. Hence there are no physically realizable processes than can cause them to either converge to, or diverge from each other. Jets accelerated by flow divergence therefore must satisfy

$$
\theta \gamma<1
$$

where $\theta$ is the opening angle of the jet. GRB jets, with inferred opening angles of a few degrees and minimum Lorentz factors of $\approx 100$, can thus not be accelerated by this process. Condition (3.12) is less constraining in the case of AGN jets, with inferred Lorentz factors in the range 3-30, but once an additional acceleration process is needed for GRB, there is no reason why it should not also operate in AGN.

\subsubsection{Conversion efficiency: artefacts}

Because of the near cancellation of terms in the magnetic acceleration, some caution is needed when interpreting results of magnetically driven flow calculations, whether they are analytic or numerical. In analytic models, innocuous looking simplifying assumptions can tip the balance in favor of one of the two terms, leading to a spurious acceleration or deceleration.

In numerical work, the unavoidable presence of numerical diffusion of field lines can cause artificial acceleration. If this diffusion is strong enough, the toroidal field can decay by annihilation across the axis. This results in a decrease of the magnetic pressure with distance along the axis, increasing the pressure gradient term in (3.4), again resulting in acceleration. The signature of such an artefact is of course that, as numerical resolution is improved, the acceleration obtained decreases. Acceleration by a decrease in magnetic energy along the flow is itself a real effect, however, if a mechanism for dissipating magnetic energy is present. I return to this in section (5).

\section{4 'Magnetic towers'}

Another picture of magnetic jets produced by a rotating, axisymmetric source is that of a 'magnetic tower'. It is sometimes presented as an intrinsically separate mechanism with its own desirable properties. It is a simplified picture of the magnetic acceleration process, in which the 
magnetic field is depicted as a cylindrical column of wound-up magnetic field. One loop of toroidal field added to the column for each rotation of the footpoints, much like a bolt rotating through a nut. The tower is assumed to be in pressure balance with an external confining medium.

The attraction of this model is that it is tractable, and visualizable on account of its axisymmetry. In addition, some of the numerical simulations look much like this picture. This the case in particular for simulations done in a cylindrical numerical grid.

\section{Ordered magnetic fields}

An ordered magnetic field is usually assumed in work on magnetocentrifugal acceleration: a field of uniform polarity threading the (inner regions of) a disk. This is sometimes chosen as a 'representative idealization' of more complicated configurations such as might result from magnetic fields generated in the disk. Such a configuration has the advantage of simplicity: all field lines anchored in the disk extend to infinity, and the flow can be a smooth function of distance from the axis. If the field is not of uniform polarity, some field lines form closed loops connecting parts of the disk surface instead of extending to infinity (cf. fig 5 in Blandford and Payne 1982), and the outflow will be patchy.

A field of uniform polarity, however, is subject to an important constraint: it cannot be created in situ by local processes in the disk. It can only exist as a consequence of either the initial conditions, or of magnetic flux entering or leaving the disk through its outer (radial) boundary. To see this formally, consider a circle of at $r=R, z=0$ (in cylindrical coordinates $r, \phi, z$ centered on the disk), where $R$ could be the outer radius of the disk, or the radial outer boundary of the computational volume. Let $S$ be a surface with this circle as its boundary, and normal vector $\mathbf{n}$, and let $\Phi=\int \mathrm{d} S \mathbf{B} \cdot \mathbf{n}$ the magnetic flux through this surface. On account of $\operatorname{div} \mathbf{B}=0, \Phi$ is independent of the choice of $S$, as long as the boundary at $r=R$ is fixed, and we can take $S$ to be in the midplane $z=0$ of the disk. With the induction equation, we have

$$
\partial_{t} \Phi=\int \mathrm{d} r \mathrm{~d} \phi r[\nabla \times(\mathbf{u} \times \mathbf{B})]_{\mathbf{z}} .
$$

With $u_{r}(0, \phi, z)=B_{r}(0, \phi, z)=0$ by symmetry of the coordinate system, this yields

$$
\partial_{t} \Phi=-\int \mathrm{d} \phi R\left[u_{z} B_{r}-u_{r} B_{z}\right],
$$

where the integrand is evaluated at $r=R$. The square bracket can be written as $u_{\perp} B_{\mathrm{p}}$, where $B_{\mathrm{p}}$ is the poloidal field $\left(B_{r}, B_{z}\right)$ and $\mathbf{u}_{\perp}$ the velocity component perpendicular to it. The RHS of (4.2) can thus be identified as the net advection of poloidal field lines through the outer boundary.

If this flux of field lines across the outer boundary vanishes, the net magnetic flux $\Phi$ through the disk is constant. It vanishes if it vanishes at $t=0$ : it can not be created by local processes in the disk, including large scale dynamos (even if these were to exist in accretion disks).

The magnetic flux through a disk is therefore a global quantity rather than a local function of conditions near the center. It depends, if not on initial conditions, on the way magnetic flux is transported through the disk as a whole. Since it is not just a function of local conditions in the disk, it acts as a second parameter in addition to the main global parameter, the accretion rate. This has an interesting observational connection: not all disks produce jets, and the ones that do, don't 
do it all the time. The possibility suggests itself that this variation is related to variations in the magnetic flux parameter of the disk (Spruit and Uzdensky 2005).

A particular attraction of ordered fields is that they can be significantly stronger than the fields produced by magnetorotational (MRI) turbulence. The energy density in MRI fields is limited to some (smallish) fraction of the gas pressure. The exact fraction achievable still appears to depend on details such as the numerical resolution, with optimistic values of order 0.1 widely quoted, while values as low as 0.001 are being reported from some of the highest resolution simulations (Fromang et al. 2007).

The strength of ordered fields, on the other hand, can be significantly higher, limited in principle only by equipartitition of magnetic energy with orbital kinetic energy, or equivalently by the balance of magnetic forces with gravity. In practice, interchange instabilities already set in when the fractional support by magnetic forces against gravity reaches a few per cent, as shown by the numerical simulations of Stehle and Spruit (2001). For a thin disk, this is substantially larger than equipartition with gas pressure. Magnetic fields of this strength actually suppress magnetorotational instability. Instead, their strength is limited by instability of the field itself rather than the shear in the orbital motion (see discussion in Stehle and Spruit).

Strong fields are also indicated by the observations of radidly varying optical emission in some sources, in particular GX 339-4 and KV UMa. As shown by Fabian et al (1982) the only realistic interpretation of this radiation is thermal synchrotron emission from a compact region near the black hole. The inferred optical depth requires very strong magnetic fields (Kanbach et al. 2001), probably larger than can be provided by MRI turbulence.

\subsection{Ordered magnetic fields in numerical simulations}

Equation (4.2) says that the net magnetic flux $\Phi$ through the disk does not change unless there is a net advection of field lines into or out of the disk boundary. This implies that the velocity field inside the disk can not create a net poloidal flux, no matter how complex or carefully construed the velocities.

A bundle of ordered magnetic flux threading a black hole, such as seen in simulations (e.g. De Villiers et al. 2005, McKinney and Gammie 2004, Hawley and Krolik 2006), can only have appeared 'in situ' by violation of $\operatorname{div} \mathbf{B}=0$. Since this is unlikely with the codes used, the flux bundles seen must have developed from flux that was already present at the start of the simulation.

The way this happens can be illustrated with the simulations of McKinney and Gammie (2004). The initial state used there is a torus of mass, with an initially axisymmetric field consisting of closed poloidal loops. The net poloidal flux through the midplane of the calculation thus vanishes: upward flux in the inner half of the torus is compensated by downward flux in the outer part. The differential rotation in the torus generates MRI turbulence causing the torus to spread quasi-viscously, the inner parts spreading towards the hole while the outer parts spread outwards. The magnetic loops share this spreading: the upward flux in the inner part spreads onto the hole, the downward flux outward.

This explains the formation of a flux bundle centered on the hole, but also makes clear that the flux of this bundle is a function of the initial conditions, and its formation the consequence of the spreading of the initial torus. The process as simulated in this way, starting with a torus close to the hole, is not really representative for conditions in an extended long-lived accretion disk. If a 
poloidal loop as in the initial conditions of present simulations were present in an actual accretion flow, the downward part of the flux would accrete onto the hole as well, canceling the flux threading the hole.

The dependence on initial is demonstrated more explicitly by some results in Machida and Matsumoto (2000b) and De Villiers et al. (2005), where initial conditions are given (a toroidal instead of a poloidal field) that resulted in very similar magnetic disk turbulence, but without an ordered Poynting flux jet.

This disagrees with suggestions that such jets would be a natural generic result of MRIgenerated magnetic fields, or even that MRI turbulence would create a net magnetic flux through the disk (e.g. McKinney \& Narayan 2007).

Existing simulations thus show that a flux bundle at the center of a disk can form from appropriate initial conditions, but leave open the question which physics would lead to such conditions. As I argue below, this need not be seen as an inconvenience. The same question may well be related to the puzzling phenomenology of X-ray states in X-ray binaries.

\subsection{Accretion of ordered magnetic fields?}

If magnetic fields of net polarity cannot be created internally in a disk, but a net polarity at the center of the disk is still considered desirable, there are two possibities:

- The field is accreted from the outside (a companion, or the interstellare medium),

- It forms by systematic separation of polarities somewhere in the disk by an as yet unknown mechanism.

The first of these has been a subject of several studies, of which the conclusions are discouraging. The model used is that of a diffusing disk, where angular momentum transport is mediated by a viscosity $v$, and the magnetic field diffuses with diffusivity $\eta$. If both result from some quasiisotropic turbulence, they are of similar magnitude.

Vertical field lines are accreted at a rate $\sim v / r$, while diffusing outward at a rate $\sim \eta / r$. In a steady state the balance between the two would yield a strong increase of field strength towards the center of the disk. The assumption of a vertical field being accreted is very unrealistic, however, since accreted field lines cannot stay vertical. In the vacuum above the disk the field lines bend away from the regions of strong field, so that the field lines make a sharp bend on passing through the disk. As shown first by van Ballegooijen (1989), the result is that magnetic flux is accreted very inefficiently. The length scale relevant for magnetic diffusion is the disk thickness $H$, rather than $r$, and diffusion correspondingly faster. The result is that accretion in a disk with $v \approx \eta$ cannot bend field lines by more than an angle $\sim H / r$ (Lubow et al. 1994), and the increase of field strength toward the disk center is negligible.

This result would seem to exclude the accretion of magnetic flux to the center of a disk in amounts significant enough to create a strong ordered field around the central mass. The observational indications for the existence of such fields are nevertheless rather compelling. An attempt to circumvent the diffusion argument above was made by Spruit and Uzdensky (2005). We appealed there to the fact that in addition to the external field to be accreted, the disk also has its own, magnetororationally generated small scale magnetic field. This field is likely to be highly inhomogeneous, with patches of strong field separated by regions of low strength. Effects like this have been reported from recent numerical simulations (Machida et al. 2000a, Fromang et al. 2007). An 
external field accreted by the disk then does not cross the disk uniformly, but through the patches of strong field. Such patches can effectively loose angular momentum through a wind, thereby beating the diffusion argument.

The ability of an accretion flow to maintain a bundle of strong field at its center, first proposed by Bisnovatyi-Kogan and Ruzmaikin (1974), is suggested by the simulations of Igumeshchev et al. (2003), and Mckinney and Gammie (2004), and others, at least for the geometrically thick flows that are most accessible to numerical simulations.

The alternative possibility of a spontaneous separation of magnetic polarities from a mixture (as generated by MRI turbulence, for example), such that a bundle of one polarity ends up near the disk center, sounds more far-fetched since it would require breaking of a fundamental symmetry of the MHD equations, which are invariant under a change of sign of the magnetic field. The apparent separation seen in numerical simulations (McKinney and Gammie 2004 and others) is rather a consequence of the initial conditions used.

\subsection{The 'second parameter' in accreting $X$-ray sources}

Previous analytic theory, as well as the recent numerical simulations, show the advantage of an ordered magnetic field near the central object in creating powerful outflows, though less effective forms of outflow associated with random magnetorotationally generated magnetic fields appear possible as well (Igumenschev et al 2003, Machida et al. 2000b). Such ordered fields may also make the puzzling behavior of X-ray binaries easier to understand.

The phenomenology of X-ray binaries (black hole and neutron star systems) includes changes in the X-ray spectrum and of the time variability properties of the X-ray emission (see contributions elsewhere in this volume). For many years the prevailing view in the interpretation of X-ray binaries has been that this phenomenology is governed by a single parameter: the accretion rate (not counting the system parameters of the binary itself).

This view was supported by the fact that the neutron star binaries showed a systematic behavior, with their spectral and timing properties ordered aproximately along a single track in colorcolor or color-intensity diagrams. It did not agree with the observations of X-ray transients (mostly black hole systems) including the 'canonical' black hole transient GU Mus. These do not conform to the movement back and forth along a single track in color-intensity diagrams expected from a single-parameter system, showing instead motion along wide and/or irregular 'loops'.

In spite of this, the phenomenology of these transients has long been interpreted as a single sequence of states along a declining accretion rate, the apparent anomalies blamed on the transient nature of the sources. Similarity of the X-ray spectrum and the properties of the time variability is evident in states of very different brightness (e.g. the 'very high' and 'intermediate' states, Rutledge et al. 1999, Belloni this volume). This provides a compelling clue that the phenomenology is not simply a function of the instantaneous accretion rate alone. Anomalies in the neutron star binaries ('parallel tracks' e.g. van der Klis 2001), though smaller in magnitude, point in the same direction.

Instead of just the instantaneous accretion rate, one could imagine that the state of the system depends also on the history of the accretion rate. This would be the case if there is a physical property of the disk causing hysteresis, such that the state is different during increasing and decreasing accretion rates, for example. Such a mechanism might be an evaporation processes depleting the 
inner regions of the disk, such that the size of the depleted zone depends on the history of the accretion rate (see review by Homan, this volume).

A more radical idea is that a true 'second parameter' is involved in the state of the accretion flow and its X-ray and timing properties. Apart from binary parameters such as the masses and orbital separation, the mass transfer rate from the companion is the only parameter determining a (steady) hydrodynamic accretion disk. In addition, in the influential standard theory of disks based on local viscosity prescriptions, the physical state at a given point of the disk is only a function of the mass flux at that point. This makes the theory much more deterministic than the properties of $\mathrm{X}$-ray transients seem to indicate.

A useful second parameter would therefore preferably be a global quantity, but it is hard to come up with plausible candidates. As argued in Spruit and Uzdensky (2005) a promising one, however, is the net flux $\Phi$ of field lines crossing the disk. As shown above, this is a truly global parameter: its value is determined only by inheritance from the initial conditions and the boundary conditions at its outer edge; it can not be changed by local processes in the disk. One could imagine, for example, that a large amount of flux would interfere with the accretion process in the inner parts of the disk, where the flux could get concentrated, and that this has something to do with the nature of the poorly understood hard X-ray state.

An second useful property of the global magnetic flux as a second parameter is the observed relation between X-ray states and the occurence of jets from X-ray binaries. If the anomalous hard state is indeed one with a high magnetic flux in the inner disk, its connection with jets would be natural since current theory strongly suggested them to be magnetically driven phenomena.

\section{Acceleration: the role of magnetic dissipation}

In 3 dimensions, the energy carried in the form of the wound-up magnetic field can decay by internal dissipation in ways that do not occur in axisymmetry, and this turns out to be a very efficient way of accelerating the flow to high Lorentz factors (Drenkhahn 2002). The fact that this has not been recognized earlier may be due to the, at first sight anti-intuitive, nature of the effect, and the emphasis on axisymmetric models in previous work.

An initially axisymmetric flow of nearly azimuthal magnetic field is bound to be highly unstable to kinking modes, especially when it is highly collimated. In a frame comoving with such a flow, the field is close to a static, almost azimuthal configuration. The details of such configurations are well known since the early days of controlled fusion research, and purely azimuthal fields are found to be unconditionally unstable. Instability reduces the energy $B_{\phi}^{2}$ of the magnetic field, and small length scales developing under the instability can lead to reconnection, further reducing the magnetic energy. The growth time of the instability is of the order of the Alfvén travel time around a loop of azimuthal field. Instability is thus more destructive in narrow jets. Dissipation of magnetic field energy into radiation by such instability has been proposed by Lyutikov and Blandford (2003) as a mechanism to power the prompt emission of Gamma-ray bursts.

Another way of dissipating magnetic energy is to start the from a non-axisymmetric rotating magnetic field. A classic example is the pulsar wind generated by a rotating neutron star with a magnetic field inclined with respect to the rotation axis. If the asymmetry strong enough, the azimuthal field in the outflow will change sign on a length scale $L \sim \pi v / \Omega$ (a 'striped wind', Kennel 
and Coroniti 1984), where $\Omega$ is the rotation rate of the source, and $v$ the speed of the outflow. For a relativistic outflow, $L$ is of the order of the light cylinder radius of the rotator. This is generally quite small compared with the distances traveled by the flow. Dissipation of magnetic energy by reconnection of field lines can be very efficient on such short length scales.

The effect of dissipation of magnetic energy on the flow is dramatic. Where in the absence of dissipation, the balance between pressure gradient and curvature force impedes the acceleration and the conversion of Poynting flux, reducing the magnetic pressure along the path of the flow causes the balance to shift immediately in favor of the pressure gradient (see eq. 3.4), resulting in acceleration. This effect is extremely efficient in particular in GRB outflows, where the largest Lorentz factors are required (Drenkhahn 2002).

It may seem strange that one can convert Poynting flux into kinetic energy by 'throwing away' magnetic energy. The magnetic energy carried by the flow accounts for only half of the Poynting flux, however. The Poynting flux in MHD is $\mathbf{v}_{\perp} B^{2} / 4 \pi$, where $\mathbf{v}_{\perp}$ is the velocity component perpendicular to $\mathbf{B}$. This is twice the rate of advection of magnetic energy $B^{2} / 8 \pi$. The other half is accounted for by the work done by the central engine on the magnetic pressure of the outflow.

This is entirely analogous to the case of a steady hydrodynamic flow, where the energy balance is expressed by the Bernoulli function. The relevant thermal energy in that case is the enthalpy, the sum of the internal energy (equivalent to $B^{/} 8 \pi$ in our case) and the pressure (also equivalent to $B^{\prime} / 8 \pi$ ). The same acceleration will take place in a hydrodynamic flow in which internal energy is taken away (for example by radiation) along the flow. For a more extended discussion of this point see Spruit and Drenkhahn (2004).

This mechanism does not require a diverging opening angle of the flow. If the dissipation is due to magnetic instabilities, it works best at high degrees of collimation; if due to reconnection in a intrinsically nonaxisymmetric flow it works independent of the degree of collimation (Drenkhahn 2002). It may well be one of the main factors determining the asymptotic flow speed in many jets (Drenkhahn and Spruit 2002, Giannios and Spruit 2006). However, it operates at distances well beyond the Alfvén radius of the flow, distances that are not covered by present simulations with focus on the region around the black hole (cf. the discussion on length scales in section 2 above).

\section{Jet collimation}

The main purpose of this section is to remind the reader of the problems with the concept of magnetic 'hoop stress' as collimating a jet. The notion that a coiled magnetic field, as in the outflow from a magnetic rotator, will confine itself by hoop stresses is incorrect, at least in the way in which it is presented in most work, observational as well as numerical.

A magnetic field is globally expansive, corresponding to the fact that it represents a positive energy density. That is, a magnetic field can only exist if there is an external agent to take up the magnetic stresses. In laboratory fields, this agent is the current carrying coil. The magnetic stresses exterted by the field on them are the limiting factor in the field strength obtainable.

A well-known theorem, particularly useful in the astrophysical context is the "vanishing force free field' theorem. A magnetic field in on its own, i.e. without other forces in the equation of motion, must be force free, $(\nabla \times \mathbf{B}) \times \mathbf{B}=\mathbf{0}$. If a field is force free everywhere and finite (i.e. 
vanishing sufficiently fast at infinity), it vanishes identically (for proofs see, e.g. Roberts 1967, Mestel 1999, Kulsrud 2006).

In the case of magnetic jets, this means that they can exist only by virtue of a surface that takes up the stress of the magnetic field. In most numerical simulations this is the external medium surrounding the flow, assumed as part of the physical model (as in the 'magnetic towers') or simply to ease numerical problems with low gas densities. Consider the boundary between the magnetic field ('jet') and the field free region around it in such a calculation. Pressure balance at this boundary is expressed by

$$
B_{\mathrm{p}}^{2}+B_{\phi}^{2}=P_{\mathrm{e}}
$$

where $\mathbf{B}_{\mathrm{p}}=\left(B_{r}, B_{z}\right)$ is the poloidal field and $B_{\phi}$ the azimuthal component, $P_{\mathrm{e}}$ the external pressure, while the internal pressure has been neglected without loss of generality. For a given poloidal field configuration (i.e. shape and magnetic flux of the jet), the presence of an azimuthal field component increases the pressure at the boundary. Everything else being equal, this will cause the jet to expand, in spite of curvature forces acting in the interior. Confusion about the role of curvature force in this context is old. For a discussion with detailed examples see Parker (1979, Chapter 9, esp. pp170-171). The azimuthal component can cause constriction to the axis, but only in a part of the volume, around the axis itself for example, but this part can not live on its own. It is surrounded by a continuation of the field out to a boundary where the stress of the entire configuration can be taken up. These facts can probably be recognized easily in existing numerical simulations.

Since magnetic jets do not collimate themselves, an external agent has to be involved. The location of the agent is plausibly somewhere near the source of the jet, but in view of the large range in length scales (see above), this still leaves considerable room. A constraint can be derived from the observed opening angle $\theta_{\infty}$. Once the flow speed has a Lorentz factor $\gamma>1 / \theta_{\infty}$, the different directions in the flow are out of causal contact and the opening angle does not change any more (at least not until the jet slows down again by interaction with its environment). Turning this around, collimation must have taken place at a location where the Lorentz factor was still less than $1 / \theta_{\infty}$.

The responsible agent somehow must be connected with the accretion disk (especially in microquasars where there is essentially nothing else around). One suggestion (Bogovalov and Tsinganos 2005) is that the observed jet is confined by a slower outflow from the accretion disk. This can be tested in principle by detection of such outflows. Another possibility (Spruit et al. 1997) is that the ordered magnetic field at the disk center that launches the jet also exists, with declining strength, at larger distances in disk. If the strength of this field scales with the gas pressure in the disk, one finds that the shape of the field lines above the disk naturally have a nearly perfectly collimating shape. The presence and absence of well-defined jets at certain X-ray states would then be related to the details of how ordered magnetic fields are accreted through the disk (cf section 4.2).

Near the center, the accretion can be in the form of an ion-supported flow (with ion temperatures near virial) which is geometrically thick $(H / r \approx 1)$. Jets launched in the central 'funnel' of such a disk are confined by the surrounding thick accretion flow. As shown by current numerical simulations, this can lead to a fair degree of collimation, though collimation to angles of a few 
degrees and less as observed in some sources will probably require an additional mechanism.

\section{References}

[1] Bains, I., Richards, A. M. S., Gledhill, T. M., \& Yates, J. A. 2004, MNRAS, 354, 529

[2] Bisnovatyi-Kogan, G. S., \& Ruzmaikin, A. A. 1974, Ap\&SS, 28, 45

[3] Bisnovatyi-Kogan, G. S., \& Ruzmaikin, A. A. 1976, Ap\&SS, 42, 401

[4] Blandford, R. D., \& Payne, D. G. 1982, MNRAS, 199, 883

[5] Bogovalov, S., \& Tsinganos, K. 2005, MNRAS, 357, 918

[6] Daigne, F., \& Drenkhahn, G. 2002, A\&A, 381, 1066

[7] De Villiers, J.-P., Hawley, J. F., Krolik, J. H., \& Hirose, S. 2005, ApJ, 620, 878

[8] Drenkhahn, G. 2002, A\&A, 387, 714

[9] Drenkhahn, G., \& Spruit, H. C. 2002, A\&A, 391, 1141

[10] Fabian, A. C., Guilbert, P. W., Motch, C., Ricketts, M., Ilovaisky, S. A., \& Chevalier, C. 1982, A\&A, 111, L9

[11] Fromang, S. Papaloizou, J.C.B. Lesur, G. Heinemann, T. 2007, arXiv:0705.3622v1 [astro-ph]

[12] Goldreich, P., \& Julian, W. H. 1970, ApJ, 160, 971

[13] Giannios, D., \& Spruit, H. C. 2006, A\&A, 450, 887

[14] Hawley, J. F., \& Krolik, J. H. 2006, ApJ, 641, 103

[15] Holzer, T.E., 1977, J. Geophys. Res., 82, 23

[16] Hutawarakorn, B., Cohen, R. J., \& Brebner, G. C. 2002, MNRAS, 330, 349

[17] Igumenshchev, I. V., Narayan, R., \& Abramowicz, M. A. 2003, ApJ, 592, 1042

[18] Kanbach, G., Straubmeier, C., Spruit, H. C., \& Belloni, T. 2001, Nature, 414, 180

[19] Kennel, C. F., \& Coroniti, F. V. 1984, ApJ, 283, 710

[20] Kulsrud, R., 2006, Plasma Physics for Astrophysics, Princeton University Press

[21] Lubow, S. H., Papaloizou, J. C. B., \& Pringle, J. E. 1994, MNRAS, 267, 235

[22] Lyutikov, M., \& Blandford, R. 2003, arXiv:astro-ph/0312347

[23] Machida M., Hayashi M. R., Matsumoto R., 2000a, ApJ, 532, L67

[24] Machida, M., \& Matsumoto, R. 2000b, ArXiv Astrophysics e-prints, arXiv:astro-ph/0009004

[25] Matsumoto, R., Matsuzaki, T., Tajima, T., \& Shibata, K. 2001, Astrophysics and Space Science Library, 263, 247

[26] McKinney, J. C., \& Gammie, C. F. 2004, ApJ, 611, 977

[27] McKinney, J. C., \& Narayan, R. 2007, MNRAS, 375, 513

[28] Mestel, L., 1999, Stellar magnetism, Oxford : Clarendon, (International series of monographs on physics )

[29] Michel, F. C. 1969, ApJ, 158, 727 
[30] Ogilvie, G. I., \& Livio, M. 2001, ApJ, 553, 158

[31] Parker, E.N., 1979, Cosmical Magnetic Fields, Clarendon Press, Oxford

[32] Phinney, E. S. 1983, Ph.D. Thesis, University of Cambridge, UK (unpublished)

[33] Rutledge, R. E., et al. 1999, ApJS, 124, 265

[34] Spruit, H. C. 1996, ArXiv Astrophysics e-prints, arXiv:astro-ph/9602022

[35] Spruit, H. C., Foglizzo, T., \& Stehle, R. 1997, MNRAS, 288, 333

[36] Spruit, H. C., \& Drenkhahn, G. D. 2004, Astronomical Society of the Pacific Conference Series, 312, 357

[37] Spruit, H. C., \& Uzdensky, D. A. 2005, ApJ, 629, 960

[38] Stehle, R., \& Spruit, H. C. 2001, MNRAS, 323, 587

[39] van Ballegooijen, A. A. 1989, Astrophysics and Space Science Library, Kluwer, 156, 99

[40] van der Klis, M. 2001, ApJ, 561, 943

[41] Vlemmings, W. H. T., van Langevelde, H. J., \& Diamond, P. J. 2005, A\&A, 434, 1029 